\title{
Alcohol intolerance associated with Hodgkin lymphoma
}

\author{
Andrew J. Bryant MD, John H. Newman MD
}

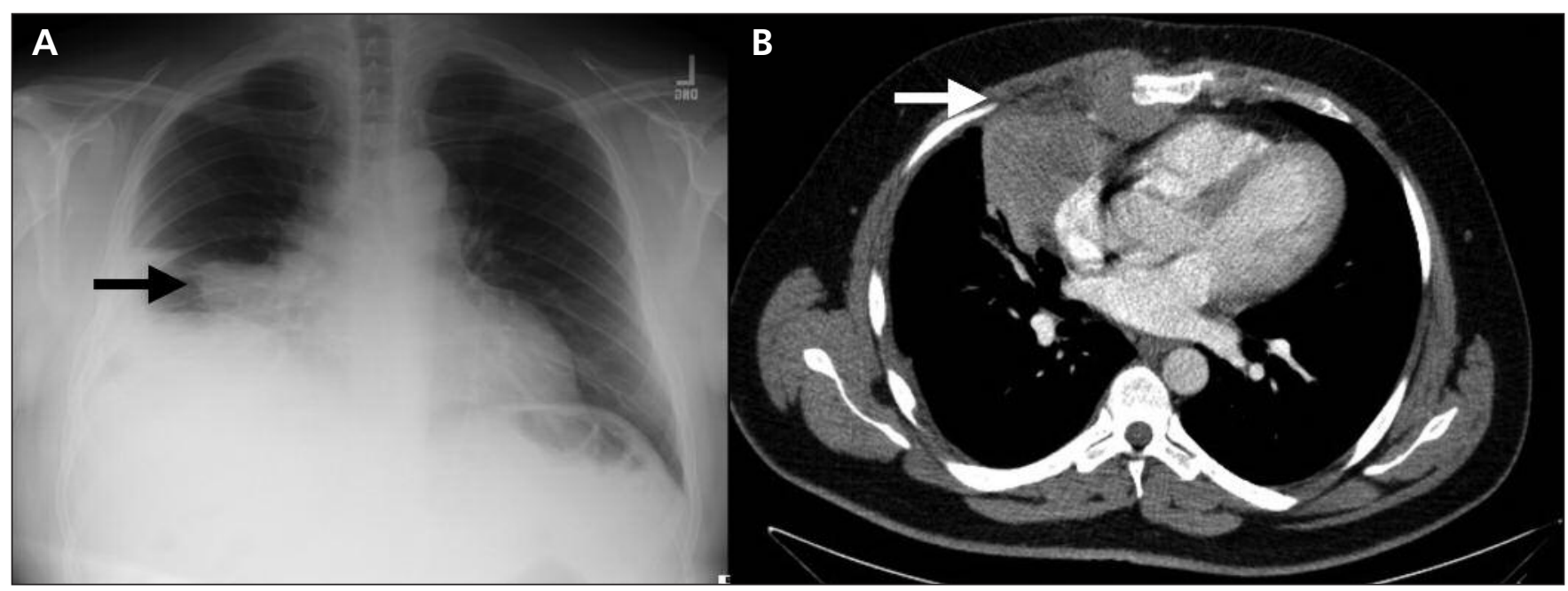

Figure 1: (A) Radiograph of the chest showing a perihilar mass in the right lung of a 31-year-old man (black arrow). (B) Computed tomography showing cardiophrenic adenopathy with invasion of the right anterior chest wall and associated sternal erosion (white arrow).

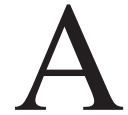

31-year-old man presented to our clinic with severe chest pain that began minutes after ingesting 2-3 sips of alcohol. This reaction had been occurring for the previous 3 months. The patient felt no pain when swallowing other liquids or solids, and his pain was relieved with low doses (400$600 \mathrm{mg}$ ) of ibuprofen.

In addition to the pain in his chest, the patient had periodic fevers, night sweats and general malaise. Imaging of the patient's chest showed a perihilar mass in the right lung, with cardiophrenic adenopathy invading the chest wall and erosion of the sternum (Figure 1A and 1B). A biopsy of an enlarged right paratracheal lymph node showed many large abnormal cells with bilobed nuclei, consistent with Reed-Sternberg cells, leading us to a diagnosis of Hodgkin lymphoma. Treatment of the cancer eventually resulted in full remission of the disease, and the patient's alcohol-related chest pain resolved soon after the first round of chemotherapeutic drugs.

The differential diagnosis of alcohol intolerance includes Hodgkin lymphoma, carcinoid syndrome and mutations of the gene encoding alcohol dehydrogenase 2 (most commonly found in Asian populations). The symptom is also associ- ated with the use of disulfiram and metronidazole. In a 1966 review describing 747 patients with alcohol-induced pain associated with neoplastic disease, Hodgkin lymphoma accounted for $40 \%$ of cases. ${ }^{1}$ A 1983 study estimated the incidence of alcohol intolerance in Hodgkin lymphoma at $1.5 \%-5 \% .^{2}$

The pathogenesis of alcohol-related pain in Hodgkin disease is poorly understood and is thought to be related to vasodilatation within the lymph node capsule following exposure to ethanol. In our patient's case, because alcoholinduced pain was relieved with ibuprofen (a cyclooxygenase inhibitor), we speculate that a prostaglandin-mediated process may have been involved. ${ }^{3,4}$ It is not known whether alcohol intolerance confers any prognostic relevance for patients with Hodgkin lymphoma.

\section{References}

1. Brewin TB. Alcohol intolerance in neoplastic disease. $B M J$ 1966;2:437-41

2. Bobrove AM. Alcohol-related pain in Hodgkin's disease. West $J$ Med 1983;138:874-5.

3. Meagher EA, Barry OP, Burke A, et al. Alcohol-induced generation of lipid peroxidation products in humans. J Clin Invest 1999;104:805-13.

4. Albano E. Alcohol, oxidative stress and free radical damage. Proc Nutr Soc 2006;65:278-90.
Competing interests: None declared.

This article has been peer reviewed.

Affiliation: From the Department of Internal Medicine, Vanderbilt University School of Medicine, Nashville, Tenn.

Correspondence to: Andrew Bryant, andrew.bryant @ vanderbilt.edu

CMAJ 2013. DOI:10.1503 /cmaj.120974 